\title{
High-temperature expansion in self-consistent-field theory of spin-crossover compounds
}

\author{
Iu.V. Gudyma, Ye.M. Daskal \\ Chernivtsi National University, Department of General Physics, 58012 Chernivtsi, Ukraine \\ E-mail: yugudyma@gmail.com
}

\begin{abstract}
In the paper, spin-crossover compounds were examined from the viewpoint of perturbation theory for self-consistent field. The research carried out in the framework of the Ising-like model for these compounds. We have obtained the first four moments for the spin state distribution function in contact with environment in a narrow temperature interval.
\end{abstract}

Keywords: molecular bistability, Ising-like model, self-consistent field, distribution function.

Manuscript received 05.07.11; revised manuscript received 10.08.11; accepted for publication 14.09.11; published online 30.11.11.

\section{Introduction}

Molecular bistability is a property of molecular system to evolve from one stable state to another stable state in response to external influence. Spin-crossover phenomenon is the best example of molecular and supramolecular bistability [1]. Spin-crossover complexes of transition metal ions having $\mathrm{d}^{4}-\mathrm{d}^{7}$ electronic configuration and might be characterized by two lowlying electronic states of different spin-multiplicity: the low-spin (LS) state with a maximum number of paired electrons and the high-spin (HS) state with a maximum number of unpaired $d$-electrons (See Fig. 1).

Compounds based on $\mathrm{Fe}^{2+}\left(3 \mathrm{~d}^{6}\right), \mathrm{Fe}^{3+}\left(3 \mathrm{~d}^{5}\right)$ and $\mathrm{Co}^{2+}\left(3 \mathrm{~d}^{7}\right)$ are the most common transition-metal ions for which the spin-state interconversion has been observed [2]. We focused on the consideration of spin-crossover compounds of iron (II) with the coordination number 6 because they have the widest practical application. Ions of all these materials can exist in one of two states, determined by different spin multiplicity, depending on the value of the ligand field $\Delta$. If $\Delta$ is greater than the average energy of spin pairs $P, d$-electrons try to occupy the lower orbital level: compound will remain in a lowspin phase where the spin state of $\mathrm{Fe}^{2+}\left(3 \mathrm{~d}^{6}\right)$ atoms are ${ }^{1} A_{1 g}$. If $\Delta$ is less than the energy of $P$, due to the Hund first rule, ions exist in a high-spin state ${ }^{5} T_{2 g}$. The electronic configurations of $\mathrm{Fe}^{2+}\left(3 \mathrm{~d}^{6}\right)$ states in these cases are presented in Fig. 2. When $\Delta$ and $P$ are the values of the same order as occurs for $\Delta$ at the point of intersection $\Delta_{c}$, spin transitions from one state to another can be controlled by external influences such as temperature, pressure and light irradiation. Thus, the spin transition results from an interaction-induced interplay between the valence electrons on the ligands and $d$-electrons in the lower energy shells located near the $\mathrm{Fe}^{2+}\left(3 \mathrm{~d}^{6}\right)$ core [3].

These states are determinate numerical: $\mathrm{S}=0$ for diamagnetic LS state and $\mathrm{S}=2$ for paramagnetic HS state. Therefore, we can say about stable $(\mathrm{S}=0)$ and metastable $(\mathrm{S}=2)$ states. This system can be considered by the instrumentality of the Ising-like model. This microscopic model was developed in the Wajnflasz pioneering works [4]. For the first time, the Ising-like model for treating nearest-neighbour interactions in spincrossover compounds was proposed Bouseksou et al. [5]. This model was successful in simulating a transition of the steady state of the Fe(II) metal compounds [6-11]. Also, for these materials many efforts have been devoted to understand theoretically spin transition in phenomenological models [12-14]. In this paper, we adopt high-temperature expansion in the field theory to microscopic Ising-like model of spin-crossover compounds.

\section{Model Hamiltonian}

It is easy to analyze the spin-crossover compounds by using for their description the Ising-like model and instrumentality of Glauber stochastic dynamics for processes of spin states changing in contact with the 


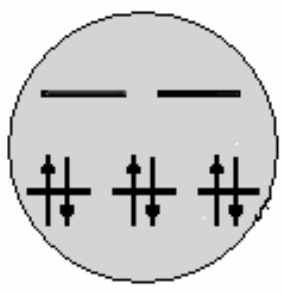

LS

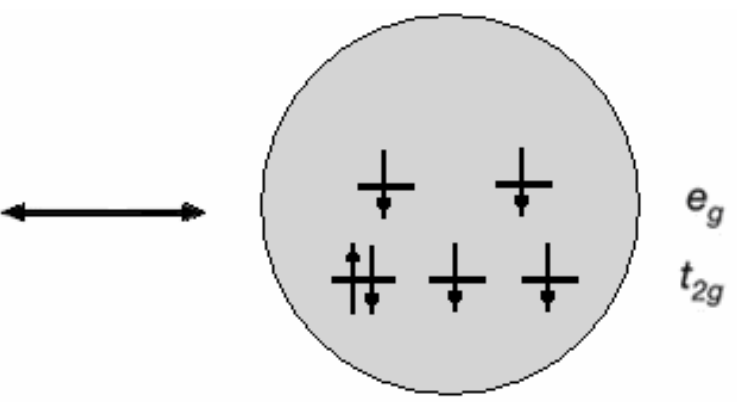

HS

Fig. 1. Spin-crossover process for $\mathrm{Fe}^{2+}\left(3 \mathrm{~d}^{6}\right)$.

environment. In the two-level scheme, the Ising-like model represents molecular states of spin-crossover material describing a fictitious spin operator $s_{i}$ with eigenvalues \pm 1 , with respective degeneracies $g_{\mathrm{HS}}$ and $g_{\text {LS }}$. The degeneracies of the eigenvalues are such that $g_{\mathrm{HS}} \neq g_{\mathrm{LS}}$, because of the different electronic and vibrational properties of the spin states. The values +1 and -1 associated with the high-spin and low-spin states of $i-$ th molecule, respectively. So, we can write the Hamiltonian of the nearest-neighbor Ising model:

$$
\mathcal{H}=-\frac{1}{2} J \sum_{\langle i j\rangle} s_{i} s_{j}+\sum_{i}\left(\Delta-\frac{k T}{2} \ln g\right) s_{i} .
$$

Let us use the designation

$$
\left(\Delta-\frac{k T}{2} \ln g\right)=H \text {. }
$$

Here, $H$ is the effective intramolecule "magnetic" field dependent on temperature, $\sum_{\langle i j\rangle}$ is the sum over the interacting neighbors (or the notation $\langle i j\rangle$ indicates that the sites $i$ and $j$ appearing in the sum are nearest neighbors), $J$ is the strength of the short-range interaction between spins, $g=g_{\mathrm{HS}} / g_{\mathrm{LS}}$ is degeneracy ratio between HS and LS, $T$ is the absolute temperature. $\Delta=E(\mathrm{HS})-E(\mathrm{LS})$ denoted energy gap (or 'fictitious field') is the enthalpy change associated with the $\mathrm{LS} \rightarrow \mathrm{HS}$ conversion of one molecule. We use positive $\Delta$, so that the ground state at low temperature is the LS phase. In the case of this model, the critical properties are the short-range Ising ferromagnet. Note that in an infinite system, with no boundaries, all spins are equivalent.

The fraction of HS molecules $n_{H}$ is the real order parameter for the present model. In order to study critical behavior of spin-crossover compounds, we adopt the magnetization per spin $\langle s\rangle=2 n_{H}-1$ as the order parameter. In this model, molecular crystal is treated as a lattice of pseudospins.

\section{Perturbation theory for self-consistent field}

Every selected spin is influenced by an effective magnetic field:

$\mathcal{H}_{\text {eff }}=-\frac{1}{2} J q \sum_{j=1}^{N} s_{j}+H$,

where $q$ is the number of the nearest neighbors of the $i-$ th spin.

It means that in the mean-field approach, we can change effective field to the following important simplification:

$$
\mathcal{H}_{e f f}^{M F}=-\frac{1}{2}(N-1)^{-1} q J \sum_{j=1}^{N} s_{j}+H,
$$

where the sum concludes all $(N-1)$ points " $j$ ", what is unequal to " $i$ ".

So, we get the mean-field Hamiltonian

$$
\begin{gathered}
\mathcal{H}=-\frac{q J}{2(N-1)} \sum_{i j} s_{i} s_{j}+H \sum_{i} s_{i}= \\
\left(-\frac{q J}{2(N-1)} \sum_{j} s_{j}+H\right) \sum_{i=1}^{N} s_{i} .
\end{gathered}
$$

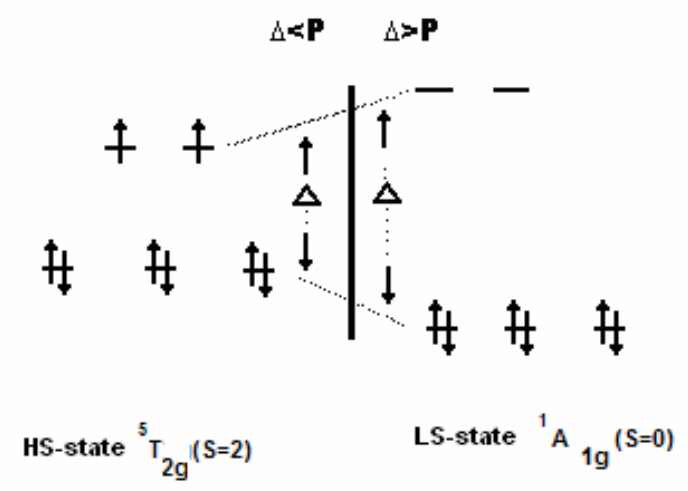

Fig. 2. Schematic representation of the electronic configurations of HS- and LS-states. 
Due to definition of the average magnetization per spin, we can write in the mean-field approximation

$$
\begin{aligned}
& \langle s\rangle=\lim _{N \rightarrow \infty} \frac{1}{N-1} \sum_{j \neq 1}^{N} s_{j}, \\
& \mathcal{H}_{\text {eff }}^{M F}=-\frac{1}{2} q J\langle s\rangle+H .
\end{aligned}
$$

On the other hand, the system has the thermal average spin value:

$$
\langle s\rangle=S p(\rho s),
$$

where $\rho=\exp (-\beta H) / S p(\exp (-\beta H))$ is the density matrix. From Eq. (8), we then find that the magnetization $\langle s\rangle$ in the mean-field approximation satisfies the self-consistency condition

$$
\langle s\rangle=\tanh \left(\beta \frac{q J}{2}\langle s\rangle-\beta H\right) .
$$

Let us denote:

$d=\frac{2 \Delta}{q J} ; \theta=\frac{2 k T}{q J} ; \frac{r}{2}=\frac{\ln g}{2}$.

As a result, we obtain:

$\langle s\rangle=\tanh \left(\frac{\langle s\rangle-\left(d-\theta \frac{r}{2}\right)}{\theta}\right)$.

With the above formulation, the condition of equilibrium in the present mean-field approach can be written as

$\theta_{e q}=\frac{2 d}{r}=\frac{4 \Delta}{q J \ln g}, 2 k T_{e q}=q J_{t h}=\frac{4 \Delta}{\ln g}$.

Fig. 3 shows the temperature dependence of the magnetization per spin for $d=1.5$ (dash), 2.5 (square), 3.5 (circle) ( $r=5$ for all the next figures). It is evident that $\langle s\rangle$ has a hysteresious behavior of temperature lower critical. Here, we find the typical $d$-dependence of $\langle s(\theta)\rangle$. That is, we find a smooth dependence for large values of $d$, and a first-order phase transition for small $d$. In the critical point, this system undergoes the secondorder phase transition.

In the spirit of the perturbation theory, we can separate the Hamiltonian to two parts $\mathcal{H}=\mathcal{H}_{0}+\mathcal{H}_{\text {int }}$, where:

$$
\begin{aligned}
& \mathcal{H}_{0}=\left(-\frac{\theta q J}{4} r+\frac{d q J}{2}\right) \sum_{i} s_{i}, \\
& \mathcal{H}_{\text {int }}=-\frac{1}{2} J \sum_{i \neq j} s_{i} s_{j} .
\end{aligned}
$$
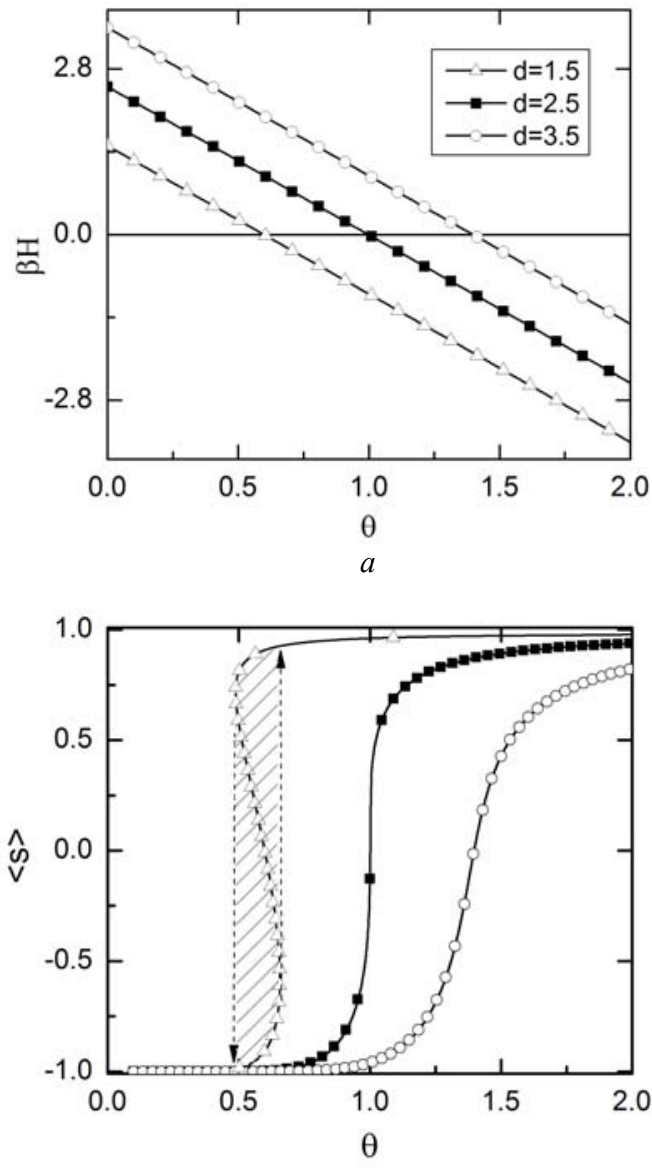

Fig. 3. Magnetic phase diagram (a) and the order parameter $\langle s\rangle$ vs temperature $\theta(\mathrm{b})$ for the ferromagnetic interaction between spins.

Our aim is to calculate correlation functions $\left\langle s_{i} s_{j} \ldots s_{l}\right\rangle$, where $\langle\ldots\rangle$ is used to denote statistical averaging the Hamiltonian $\mathcal{H}$ :

$$
\begin{aligned}
& \langle\ldots\rangle=\operatorname{Tr}\left(e^{-\beta \mathcal{H}} \ldots\right) / \operatorname{Tr} e^{-\beta \mathcal{H}}, \\
& \langle\ldots\rangle_{0}=\operatorname{Tr}\left(e^{-\beta \mathcal{H}_{0}} \ldots\right) / \operatorname{Tr} e^{-\beta \mathcal{H}_{0}} .
\end{aligned}
$$

For convenience, we can rewrite:

$$
\left\langle s_{1} s_{2} \ldots s_{l}\right\rangle=\frac{\left\langle s_{1} s_{2} \ldots s_{l} e^{-\beta \mathcal{H}_{\mathrm{int}}}\right\rangle_{0}}{\left\langle e^{-\beta \mathcal{H}_{\mathrm{int}}}\right\rangle_{0}} .
$$

For the selected point, the average spin value is given by

$$
\begin{aligned}
& \left\langle s_{1}\right\rangle_{0}=\operatorname{Tr}\left(s_{1} e^{y_{0} s_{1}}\right) / \operatorname{Tr} e^{y_{0} s_{1}}= \\
& =\frac{\sum_{i} s_{i} e^{y_{0} s_{i}}}{\sum_{i} e^{y_{0} s}}=\frac{e^{y_{0}}-e^{-y_{0}}}{e^{y_{0}}+e^{-y_{0}}}=\operatorname{th}\left(y_{0}\right) .
\end{aligned}
$$


$y_{0} \equiv-\frac{2}{\theta q J}\left(\frac{d q J}{2}-\frac{\theta q J}{4} r\right)=-\frac{d}{\theta}+\frac{1}{2} r$.

The average from every power operator $s$ we can describe, let us use the function $b\left(y_{0}\right)$ and its derivatives $b^{[n]}$ :

$\left\langle s^{N}\right\rangle=\frac{Z^{[N]}}{Z}$,

where $Z=\sum_{i} e^{y s_{i}}$.

Due to $b=\frac{Z^{\prime}}{Z}$, we get:

$\left\langle s^{[N]}\right\rangle=\frac{1}{Z}(b Z)^{[N-1]}$.

According to Leibniz equation, we obtain

$(f \cdot g)^{[N]}=\sum_{k=0}^{n} C_{n}^{k} f^{[n-k]} g^{[k]}$,

where $C_{n}^{k}=\frac{n !}{k !(n-k) !}$ are binomial coefficients.

For our case, we have recurrence relations for the high-temperature expansion

$$
\left\langle\sigma^{N}\right\rangle_{0}=\sum_{n=0}^{N-1} \frac{(N-1) !}{n !(N-1-n) !} b^{[n]}\left\langle\sigma^{N-n-1}\right\rangle_{0} .
$$

So, for the particular cases, we have first four moments

$\langle s\rangle_{0}=b$,

$\left\langle s^{2}\right\rangle_{0}=b^{2}+b^{\prime}$,

$\left\langle s^{3}\right\rangle_{0}=b^{3}+3 b^{\prime} b+b^{\prime \prime}$,

$\left\langle s^{4}\right\rangle_{0}=b^{4}+6 b^{\prime} b^{2}+3 b^{\prime 2}+4 b^{\prime \prime} b+b^{\prime \prime \prime}$.

At the following step, we rewrite a typical term of Hamiltonian (13), (14) as

$$
\begin{aligned}
& \mathcal{H}_{0}=\left(\frac{d q J}{2}-\frac{\theta q J}{4} r-\frac{1}{2} J \sum_{j \neq i}\left\langle s_{j}\right\rangle\right) \sum_{i} s_{i}, \\
& \mathcal{H}_{\text {int }}=-\frac{1}{2} J \sum_{i \neq j}\left(s_{i}-\left\langle s_{i}\right\rangle\right)\left(s_{j}-\left\langle s_{j}\right\rangle\right) .
\end{aligned}
$$

The term $\mathcal{H}_{\text {int }}$ describing of fluctuations in magnetization will be small compared to the $\mathcal{H}_{0}$ in the limit $N \rightarrow \infty$. The latter equations are the starting point for thermodynamic perturbation theory. The mean field model is the reference or zeroth-order system. We assume that fluctuations about the mean field energy are small. Thus, this approach allows for the Ising-type model taking into account correlation effects.

In this approach, the mean model energy is given by

$$
y=-\beta \mathcal{H}=-\frac{d}{\theta}+\frac{1}{2} r+\frac{\langle s\rangle}{\theta} .
$$

Recall that the distribution can be described by moments of random variables. For our case we have obtained first four moments:

$$
\begin{aligned}
& \langle s\rangle=\tanh \left(\frac{1}{2} r-\frac{d}{\theta}+\frac{\langle s\rangle}{\theta}\right), \\
& \left\langle s^{2}\right\rangle=\tanh ^{2}\left(\frac{1}{2} r-\frac{d}{\theta}+\frac{\langle s\rangle}{\theta}\right)+\frac{1}{\theta} \sec h^{2}\left(\frac{1}{2} r-\frac{d}{\theta}+\frac{\langle s\rangle}{\theta}\right),
\end{aligned}
$$

$\left\langle s^{3}\right\rangle=\tanh ^{3}\left(\frac{1}{2} r-\frac{d}{\theta}+\frac{\langle s\rangle}{\theta}\right)+$

$+\frac{3}{\theta} \sec h^{2}\left(\frac{1}{2} r-\frac{d}{\theta}+\frac{\langle s\rangle}{\theta}\right) \tanh \left(\frac{1}{2} r-\frac{d}{\theta}+\frac{\langle s\rangle}{\theta}\right)-$

$-2\left(\frac{1}{\theta}\right)^{2} \operatorname{sech} h^{2}\left(\frac{1}{2} r-\frac{d}{\theta}+\frac{\langle s\rangle}{\theta}\right) \tanh \left(\frac{1}{2} r-\frac{d}{\theta}+\frac{\langle s\rangle}{\theta}\right)$,

$\left\langle s^{4}\right\rangle=\tanh ^{4}\left(\frac{1}{2} r-\frac{d}{\theta}+\frac{\langle s\rangle}{\theta}\right)+$

$+\frac{6}{\theta} \sec h^{2}\left(\frac{1}{2} r-\frac{d}{\theta}+\frac{\langle s\rangle}{\theta}\right) \tanh ^{2}\left(\frac{1}{2} r-\frac{d}{\theta}+\frac{\langle s\rangle}{\theta}\right)+$

$+3\left(\frac{1}{\theta}\right)^{2} \sec h^{4}\left(\frac{1}{2} r-\frac{d}{\theta}+\frac{\langle s\rangle}{\theta}\right)-8\left(\frac{1}{\theta}\right)^{2} \times$

$\times \sec h^{2}\left(\frac{1}{2} r-\frac{d}{\theta}+\frac{\langle s\rangle}{\theta}\right) \tanh ^{2}\left(\frac{1}{2} r-\frac{d}{\theta}+\frac{\langle s\rangle}{\theta}\right)+$

$+2\left(\frac{1}{\theta}\right)^{3} \sec h^{2}\left(\frac{1}{2} r-\frac{d}{\theta}+\frac{\langle s\rangle}{\theta}\right) \times$

$\times\left(2 \tanh \left(\frac{1}{2} r-\frac{d}{\theta}+\frac{\langle s\rangle}{\theta}\right)-\operatorname{sech} h^{2}\left(\frac{1}{2} r-\frac{d}{\theta}+\frac{\langle s\rangle}{\theta}\right)\right)$.

From Eqs (27) and (28), we obtain for the variance, the skewness and the kurtosis, respectively,

$$
\begin{aligned}
& \sigma^{2}=\mu_{2}=\langle s\rangle^{2}-\left\langle s^{2}\right\rangle=\frac{1}{\theta} \sec h^{2}\left(\frac{1}{2} r-\frac{d}{\theta}+\frac{\langle s\rangle}{\theta}\right), \\
& \text { asym }=\frac{\mu_{3}}{\sigma^{3}}=\frac{\left\langle s^{3}\right\rangle}{\left[\frac{1}{\theta} \sec h^{2}\left(\frac{1}{2} r-\frac{d}{\theta}+\frac{\langle s\rangle}{\theta}\right)\right]^{3 / 2}}, \\
& \text { excess }=\frac{\mu_{4}}{\sigma^{4}}-3=\frac{\left\langle s^{4}\right\rangle}{\left[\frac{1}{\theta} \operatorname{sech}^{2}\left(\frac{1}{2} r-\frac{d}{\theta}+\frac{\langle s\rangle}{\theta}\right)\right]^{2}}-3
\end{aligned}
$$

The second, third and fourth moments will be considered by us in the field where temperature is higher than the critical temperature.

(C) 2011, V. Lashkaryov Institute of Semiconductor Physics, National Academy of Sciences of Ukraine 


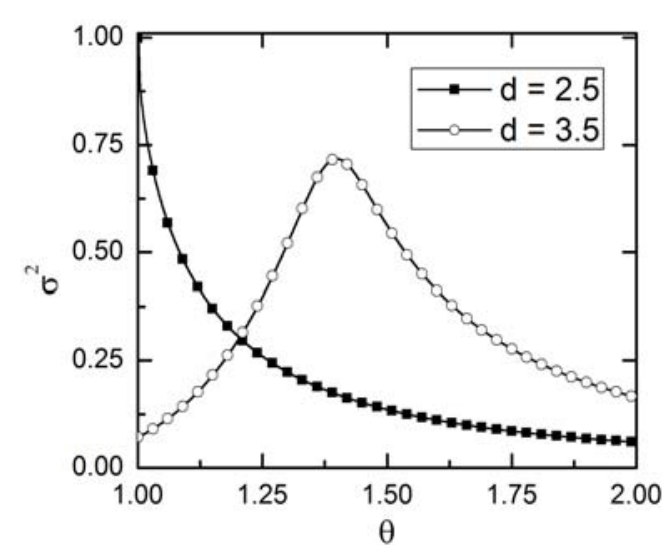

Fig. 4. Variance as a function of temperature.
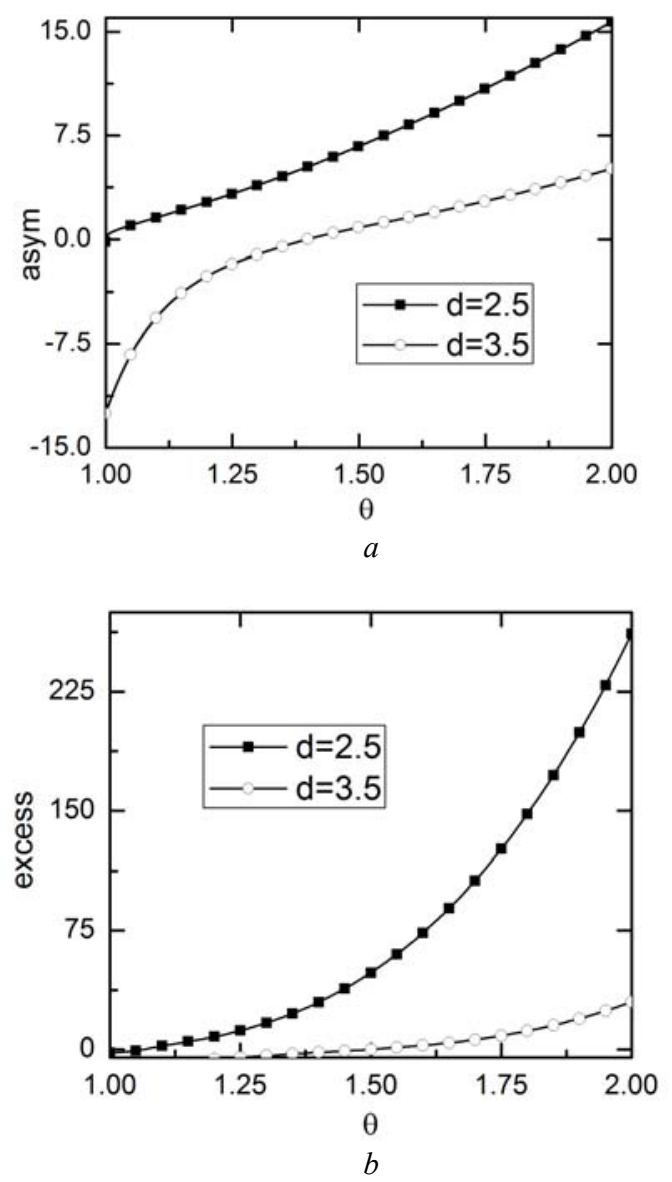

Fig. 5. Plots of the skewness (a) and kurtosis (b) of the spin probability density.

Fig. 4 shows the mean variance obtained by solution of Eq. (33) for various $d$. The stationary variance of the corresponding probability distribution is finite. In the critical point, we see that the variance decreases as the temperature increases. The variance exhibits a single-peak shape as shown in Fig. 4 with the curve at $d=3.5$ above the critical point.

We calculated also the skewness (34) that characterizes the degree of asymmetry of distribution function of fictitious spin $s$ and the kurtosis (35) that characterizes the degree of peakedness of this distribution function. Because these values are equal to zero identically to a Gaussian distribution, one can consider the skewness and kurtosis as appropriate measures of deviation of distribution from a Gaussian shape. In the high temperature case, the data show that the probability distribution function slowly approaches to Gaussian.

\section{Concluding remarks}

Summarizing, in high-temperature approach we have derived and analyzed the equations for four first moments of the distribution function of the net magnetization in spin-crossover compounds. It allows us to reduce the distribution function and provide the behavior of the molecules in spin-crossover materials under the external influences. In order to characterize the difference of the spin probability density from the Gaussian density, we have calculated the skewness and kurtosis. According to this theory, one could qualitatively expect the behavior of probability distribution of magnetization per spin in spin-crossover compounds.

Our results agree with those obtained by other methods.

\section{Acknowledgements}

This work was partially supported by the project of the Ministry of Education, Science, Youth and Sports of Ukraine under Grant No 0110U000190. We would like to thank Artur Maksymov for performing the computer programming.

\section{References}

1. Transition Metal in Supramolecular Chemistry, Ed. by J.-P. Sauvage. John Wiley \& Sons, New York, 1999.

2. Spin Crossover in Transition Metal Compounds I, Ed. by P. Gütlich, H.A. Goodwin. Springer-Verlag, Berlin, 2004.

3. V. Meded, A. Bagrets, K. Fink, R. Chandrasekar, M. Ruben, F. Evers, A. Bernand-Mantel, J.S. Seldenthuis, A. Beukman, H.S.J. van der Zant, Electrical control over the Fe(II) spin crossover in a single molecule: Theory and experiment // Phys. Rev. B, 83(24), 245415 (2011).

4. J. Wajnflasz, R. Pick, Transitions "Low spin""High spin" dans les complexes de $\mathrm{Fe}^{2+} / /$ J. Phys. Colloq. France, 32, p. 537-545 (1971).

5. A. Bousseksou, J. Nasser, J. Linares, K. Boukheddaden, F. Varret, Ising-like model for the two-step spin-crossover // J. Physique, 1(2), p. 1381-1403 (1992). 
6. S. Miyashita, Y. Konishi, M. Nishino, H. Tokoro, P.A. Rikvold, Realization of the mean-field universality class in spin-crossover materials // Phys. Rev. B, 77(1), 01410 (2008).

7. C. Timm, C.J. Rye, Reentrant magnetic ordering and percolation in spin-crossover system // Phys. Rev. B, 77 (21), 214437 (2008).

8. S. Mouri, K. Tanaka, S. Bonhommeau, N.O. Moussa, G. Molnár, A. Bousseksou, Relaxation process from photoinduced states of double-step spin-crossover systems using a kinetic two-sublattice Ising-like model including intra-site coupling // Phys. Rev. B, 78(17), 174308 (2008).

9. W. Nicolazzi, S. Pillet, C. Lecomte, Photoinduced phase separation in spin-crossover materials: Numerical simulation of a dynamic photocrystallographic experiment // Phys. Rev. B, 80(13), 132102 (2009).
10. C. Chong, F. Varret, K. Boukheddaden, Evolution of self-organized domains under light in singlecrystalline $\left[\mathrm{Fe}(\mathrm{ptz})_{6}\right]\left(\mathrm{BF}_{4}\right)_{2} / /$ Phys. Rev. B, 81(1), 014104 (2010).

11. N. Klinduhov, D. Chernyshov, K. Boukheddaden, Choice of dynamics for spin-crossover systems // Phys. Rev., 81(9), 094408 (2010).

12. Iu. Gudyma, A. Maksymov, C. Enachescu, Decay of a metastable high-spin state in spin-crossover compounds: mean first passage time analysis // Eur. Phys. J. B, 78(2), p. 167-172 (2010).

13. Iu.V. Gudyma, A.Iu. Maksymov, Nonlinear stochastic relaxation dynamics in spin-crossover solid-state compounds // Semiconductor Physics, Quantum Electronics \& Optoelectronics, 13(4), p. 357-362 (2010).

14. Iu.V. Gudyma, A.Iu. Maksymov, High spin metastable state relaxation of spin-crossover solids driven by white noise // J. Phys. and Chem. Solids, 72(2), p. 73-77 (2011). 